\title{
Unicuspid Acommissural Aortic Valve with Supra-aortic Membrane: An Extremely Rare Congenital Anomaly
}

\author{
Santosh Kumar Sinha \\ LPS Institute of Cardiology. G.S.V.M. Medical College Kanpur-208002, Uttar Pradesh, India.
}

\section{Corresponding Author:}

Dr Santosh Kumar Sinha

Email: fionasan@rediffmail.com

This is an Open Access article distributed under the terms of the Creative Commons Attribution License (creativecommons.org/ licenses/by/3.0).

Received Accepted Published

May 10, 2020

September 3, 2020

June 15, 2021

\begin{abstract}
Background: Unicommissural aortic valve (UAV) is diagnosed by means of echocardiography during systole, because absence of cusp separation reveals eccentric "teardrop" opening. Case Report: A 4-month infant was refereed for evaluation of murmur at aortic area by his pediatrician. On examination, peripheral pulses were weak with diminished and delayed carotid upstroke. Transrthoracic echocardiography revealed unicuspid, unicommissural aortic valve (UAV) with moderate aortic stenosis. There was a supra-aortic membrane without any aortic leak. Conclusion: The importance of its early diagnosis lies in its risk of sudden cardiac death which can be mitigated and other associated congenital anomalies can also be corrected.
\end{abstract}

Keywords: Aortic Valve Stenosis, Echocardiography, Heart Valve Diseases, Unicuspid Aortic Valve.

\section{Introduction}

Unicommissural aortic valve (UAV), an extremely rare congenital malformation was first reported by Edwards in 1958 [1]. During embryogenesis, valve cusps, sinuses, and commissures evolves into normal aortic trileaflet valve with 3 cusps and 3 associated commissures. UAV is outcome of failure of three aortic cusps to separate before birth. It may be either isolated, or be associated with other anomalies such as patent ductus arteriosus, aortic aneurysms and coarctation of aorta $[2,3]$. Its annual incidence is $0.02 \%$ in adult population whereas $4-5 \%$ among surgical series [3].

\section{Case Report}

A 4-month infant was referred by his pediatrician for incidental detection of murmur at aortic area during routine checkup. On examination, peripheral pulses were weak with diminished and delayed carotid upstroke. There was no radio-femoral delay. Auscultation revealed normal S1 and soft
S2. A grade III/VI ejection systolic murmur with delayed peak systolic was best heard in aortic area which was radiating to neck. There was no ejection click. Jugular venous pressure was normal. Rest of clinical examination was within normal limits. Electrocardiogram was suggesting normal sinus rhythm with left ventricular hypertrophy. Transthoracic echocardiography revealed a posteriorly situated eccentric aortic valve orifice extending to annulus in systole. It was unicuspid, unicommissural aortic valve (UAV) with moderate aortic stenosis (peak pressure gradient of 48 $\mathrm{mmHg}$ ) [Fig.1]. Biventricular systolic functions were normal. There was a supra-aortic membrane without any aortic leak [Fig.2].

\section{Discussion}

Unicuspid acommissural valve is devoid any commissural or lateral attachments to aorta at level of orifice, so they have a central opening, whereas unicommissural one has one lateral commissural 
attachment to aorta at level of orifice and, in its morphology, appears as a slit-shaped structure having an eccentric orifice which could be linear (like an exclamation mark), triangular, oval, or "tear drop" shape. Therefore, they present at early age with severe aortic stenosis as orifice may be pinhole. The orifice in unicommissural UAV is larger than that in acommissural UAV, as a result these patients typically remain asymptomatic until adulthood [4]. UAV is diagnosed by means of echocardiography during systole, because absence of cusp separation reveals eccentric "teardrop" opening [5]. Another novel and rare finding in our case was supra-aortic membrane. Aortic stenosis mostly isolated or rarely with aortic regurgitation is most common presentation of UAV. It is often associated with dilatation or dissection of aorta, involving aortic root, ascending aorta, or aortic arch, because of common embryological defect (medial changes in aortic tissue). As it is an aortopathy, it has a bimodal distribution where younger one presents with aggressive form of aortic root dilation whereas older patients usually have no aortic root dilation. Surgical intervention is exercised in the form of aortic valve replacement with/or without Bental's correction. The importance of its early diagnosis lies in its risk of sudden cardiac death which can be mitigated and other associated congenital anomalies can also be corrected. In our case, as it was moderate AS, conservative treatment was advised with repeat echocardiography every 6 months.

\section{Conclusion}

Meticulous clinical examination still remains the gold standard approach in diagnosis and subsequent management of a patient. Unicuspid aortic valve (UAV) with supra-aortic membrane may present with isolated stenosis only. If aortic stenosis is severe, surgery is advisable. Repeat echocardiography is needed to follow up in case of mild to moderate stenosis.

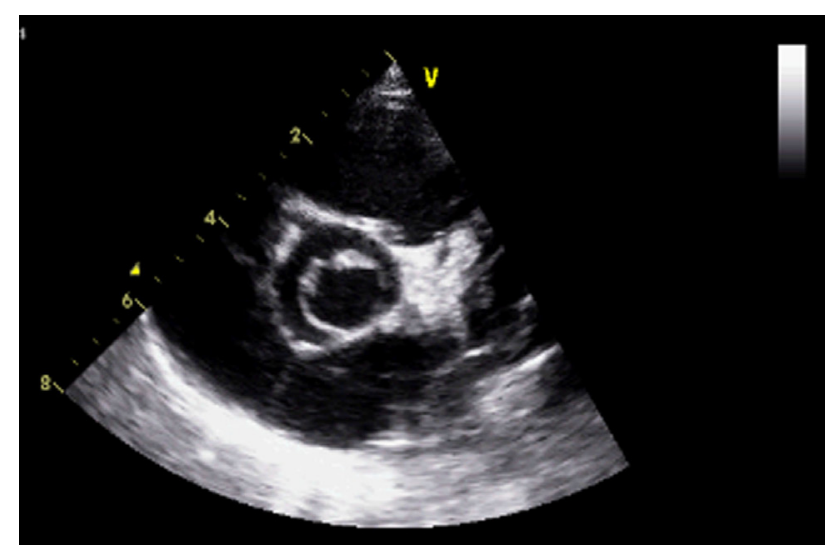

Fig.1: Transthoracic echocardiogram in parasternal short axis view showing unicuspid unicommissural valve with eccentric valvular orifice. The valve has a single leaflet as seen in systole.

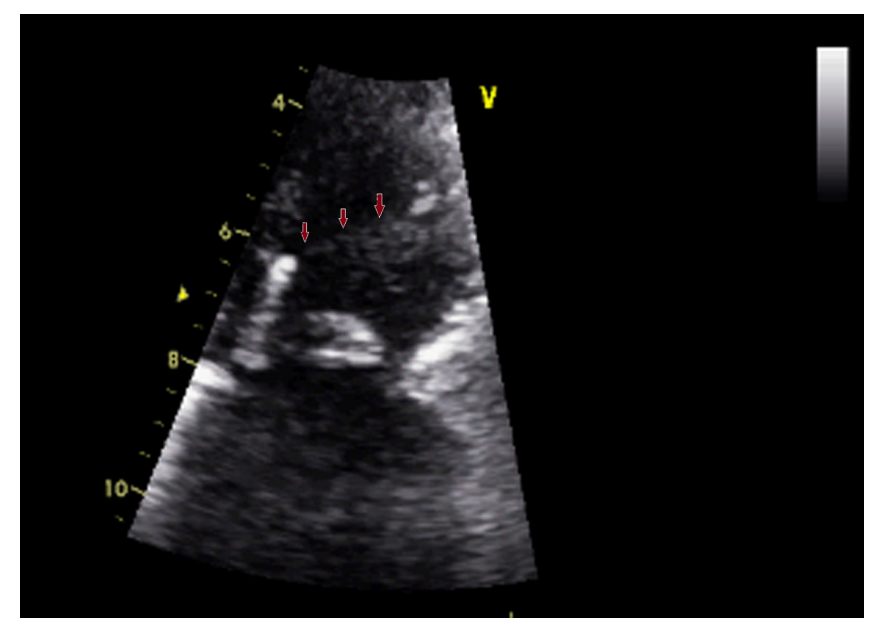

Fig.2: A supra-aortic membrane without any aortic leak.

Contributors: SKS drafted the article and was involved in patient management gave final approval of the version to be submitted, will act as a study guarantor and is responsible for all aspects of this study.

Funding: None; Competing interests: None stated.

\section{References}

1. Edwards JE. Pathologic aspects of cardiac valvular insufficiencies. AMA Arch Surg. 1958;77:634-649.

2. Ward C. Clinical significance of the bicuspid aortic valve. Heart. 2000;83:81-85.

3. Roberts WC, Ko JM. Frequency by decades of unicuspid, bicuspid, and tricuspid aortic valves in adults having isolated aortic valve replacement for aortic stenosis, with 
or without associated aortic regurgitation. Circulation. 2005;111:920-925.

4. Anderson RH. Understanding the structure of the unicuspid and unicommissural aortic valve. J Heart Valve Dis. 2003; 12:670-673.
5. Novaro GM, Mishra M, Griffin BP. Incidence and echocardiographic features of congenital unicuspid aortic valve in an adult population. J Heart Valve Dis. 2003;12:674-678. 